TRANSACTIONS OF THE

AMERICAN MATHEMATICAL SOCIETY

Volume 354, Number 8, Pages 3059-3074

S 0002-9947(02)03004-0

Article electronically published on April 2, 2002

\title{
LIGHT STRUCTURES IN INFINITE PLANAR GRAPHS WITHOUT THE STRONG ISOPERIMETRIC PROPERTY
}

\author{
BOJAN MOHAR
}

\begin{abstract}
It is shown that the discharging method can be successfully applied on infinite planar graphs of subexponential growth and even on those graphs that do not satisfy the strong edge isoperimetric inequality. The general outline of the method is presented and the following applications are given: Planar graphs with only finitely many vertices of degree $\leq 5$ and with subexponential growth contain arbitrarily large finite submaps of the tessellation of the plane or of some tessellation of the cylinder by equilateral triangles. Every planar graph with isoperimetric number zero and with essential minimum degree $\geq 3$ has infinitely many edges whose degree sum is at most 15 . In particular, this holds for all graphs with minimum degree $\geq 3$ and with subexponential growth. The cases without infinitely many edges whose degree sum is $\leq 14$ (or, similarly, $\leq 13$ or $\leq 12$ ) are also considered. Several further results are obtained.
\end{abstract}

\section{INTRODUCTION}

An infinite graph $G$ satisfies the strong edge isoperimetric inequality if there is a positive constant $h$ such that every finite vertex set $X$ has at least $h|X|$ outgoing edges. The supremum of all such constants $h$ is called the isoperimetric number of $G$. Graphs with positive isoperimetric number have many interesting properties and have been studied in relation to various other problems. For example, Dodziuk [6], Gerl [8] and Soardi [20] considered them in relation to random walks and their transience, Mohar [17, 18, 19] and Žuk [23] were interested in their spectral properties, Gromov in their hyperbolic geometric 9] and hyperbolic groups structure [10], Lyons et al. [14, 16] considered applications in theoretical physics (phase transition and percolation), Woess 22] was motivated by the study of harmonic functions, while He and Schramm [13] investigated circle packings.

It is easy to see that every finite planar graph contains a vertex of degree at most 5. This is not true for infinite planar graphs whose minimum vertex degree can be arbitrarily large. However, if an infinite planar graph has minimum degree greater than 6 , it has exponential growth and satisfies the strong isoperimetric inequality. This fact was proved by Dodziuk [6], who used the strong isoperimetric inequality to prove transience of random walks on such graphs. Extensions of Dodziuk's result were obtained by various authors in different contexts. See, e.g., Mohar [19], Soardi [20], Calogero [5], Žuk [23], Woess [22], and Baues and Peyerimhoff [3]. Another

Received by the editors March 19, 2001.

2000 Mathematics Subject Classification. Primary 05B45, 52B60, 52C20, 60J10.

Supported in part by the Ministry of Science and Technology of Slovenia, Research Project J1-0502-0101-00. 
extension is obtained in this paper, where it is proved that every planar graph with only finitely many vertices of degree $\leq 5$ and of subexponential growth contains every finite submap of $T_{0}$ or of $T_{0} / \Gamma$, where $T_{0}$ is the infinite triangular lattice $\left[6^{3}\right]$ and $\Gamma \leq \operatorname{Aut}\left(\left[6^{3}\right]\right)$ is an infinite cyclic group.

If $u v$ is an edge of a graph, the weight of $u v$ is defined as the sum of degrees of its end vertices. Kotzig [15] proved that every (finite) 3-connected planar graph contains an edge $u v$ of weight at most 13. Grünbaum and Shephard [11 extended Kotzig's theorem to graphs of periodic tilings of the plane by proving that there is an edge of weight at most 15 . Such graphs are universal covering spaces of graphs embedded in the torus. Stehling [21] extended the result of Grünbaum and Shephard to normal tilings of the plane (where all tiles are uniformly bounded). Theorems 5.1 and 5.2 and Corollary 5.5 in this paper are extensions of that result to arbitrary infinite planar graphs of minimum degree $\geq 3$ and with subexponential growth. Let us observe that the graphs considered by Grünbaum and Shephard in [1] or by Stehling [21] have quadratic growth, have only one end, and have only faces of finite length.

Moreover, we prove that every connected infinite planar graph whose isoperimetric number is zero contains infinitely many edges of weight at most 15 , and we also show that graphs without infinitely many edges of weight $\leq 14$ (or $\leq 13$ or $\leq 12$ ) contain arbitrarily large submaps of certain tessellations of the plane or the flat cylinder.

More generally, it is shown that the well-known discharging method (used, for example, in the proof of the Four Color Theorem [2, 12]) can be extended from finite planar graphs to infinite planar graphs of subexponential growth, and usually also to planar graphs whose edge isoperimetric number is zero. The main result (Theorem 3.1) and its proof give rise to explicit positive lower bounds on the isoperimetric number.

It is worth mentioning that planar graphs treated in this paper are much more general than graphs of normal tilings, since they may have any of the following properties:

(a) Growth rates are arbitrary.

(b) Vertex degrees need not be bounded.

(c) Face lengths may be unbounded and faces of infinite length may exist.

(d) The graph may have more than one end (even uncountably many are possible).

In most previous works (e.g., 3, 5, 11, 14, 21, 22, 23]), (a)-(d) are not allowed.

Graphs in this paper are simple (no loops, no multiple edges) and locally finite, i.e., finite or countably infinite with the degree of every vertex finite.

We follow standard graph theory terminology and notation. If $G$ is a graph and $v \in V(G)$, then $\operatorname{deg}_{G}(v)$ is the degree of $v$. If $G$ is embedded in the plane and $f$ is a face of $G$, then $\operatorname{deg}_{G}(f)$ denotes the length of $f$. If $X \subseteq V(G)$, then $G(X)$ is the subgraph of $G$ induced on the vertex set $X$, and $\delta X$ is the set of all edges with one endvertex in $X$ and the other end in $V(G) \backslash X$. For a positive integer $r, N_{r}(X)$ denotes the set of all vertices in $V(G) \backslash X$ whose distance from $X$ is at most $r$.

\section{STRONG ISOPERIMETRIC PROPERTY}

Let $G$ be a connected graph and let $v \in V(G)$. Denote by $B_{n}(v)$ the set of all vertices of $G$ at distance at most $n$ from $v$ (the ball of radius $n$ centered at $v$ ), and let $b_{n}(v):=\left|B_{n}(v)\right|$. If the graph $G$ is not clear from the context, we write 
$b_{n}(G, v)$, or $B_{n}(G, v)$. The graph $G$ has exponential growth (from the vertex $v$ ) if $b_{n}(v) \geq C q^{n}$ for some constants $C>0$ and $q>1$, and for every $n \geq 0$. It has polynomial growth if $b_{n}(v) \leq p(n)$, where $p(\cdot)$ is a polynomial. $G$ has subexponential growth if it does not grow exponentially. Let us observe that the type of the growth is independent of $v$, since $G$ is connected. Let

$$
\tau(G)=\liminf _{n \rightarrow \infty}\left(b_{n}(G, v)\right)^{1 / n} .
$$

If $v$ and $u$ are vertices of $G$ and $d=\operatorname{dist}(u, v)$, then $b_{n}(u) \leq b_{n+d}(v) \leq b_{n+2 d}(u)$. It follows that $\tau(G)$ does not depend on the choice of $v$. The following is an easy exercise.

Proposition 2.1. Let $G$ be a connected graph. Then $\tau(G)>1$ if and only if $G$ has exponential growth.

The (edge) isoperimetric number $h_{1}(G)$ of $G$ is the number

$$
h_{1}(G)=\inf \left\{\frac{|\delta X|}{|X|}|X \subset V(G), 0<| X \mid<\infty\right\} .
$$

Similarly, the (vertex) isoperimetric number $h_{0}(G)$ is defined as

$$
h_{0}(G)=\inf \left\{\frac{\left|N_{1}(X)\right|}{|X|}|X \subset V(G), 0<| X \mid<\infty\right\} .
$$

Since every vertex in $N_{1}(X)$ is incident with an edge in $\delta X, h_{0}(G) \leq h_{1}(G)$.

Proposition 2.2. Let $G$ be a connected graph. Then $\tau(G) \geq 1+h_{0}(G)$. If $G$ is a planar graph, then $\tau^{2}(G) \geq 1+\frac{1}{2} h_{1}(G)$.

Proof. Let $v \in V(G)$. For $n \geq 1$, let $b_{n}=b_{n}(v)$ and $s_{n}=b_{n}-b_{n-1}$. By selecting $X=B_{n-1}(G, v)$, we conclude that $s_{n}=\left|N_{1}(X)\right| \geq h_{0}(G)|X|=h_{0}(G) b_{n-1}$. This easily implies that $b_{n} \geq\left(1+h_{0}(G)\right)^{n}$. Consequently, $\tau(G) \geq 1+h_{0}(G)$.

To prove the second inequality, let $X$ be as above and let $A=N_{1}(X)$ and $B=N_{1}(X \cup A)$. Then $A \cup B$ and the edges joining $A$ and $B$ form a bipartite planar graph. Euler's formula and standard counting arguments imply that this graph has less than $2|A \cup B|=2 s_{n}+2 s_{n+1}$ edges. On the other hand, the edge set of this graph is $\delta(X \cup A)$ and hence its cardinality is at least $h_{1}(G)|X \cup A|$. As a consequence, $h_{1}(G) b_{n} \leq 2\left(b_{n+1}-b_{n-1}\right)$. Therefore, $b_{n} \geq\left(\frac{h_{1}(G)}{2}+1\right)^{(n-1) / 2}$. This implies that $\tau^{2}(G) \geq \frac{h_{1}(G)}{2}+1$.

There are planar graphs $G$ such that $h_{0}(G)=h_{1}(G)=0$ and $\tau(G)>1$, and there are planar graphs for which $h_{0}(G)=0$ and $h_{1}(G)>0$ (and hence also $\tau(G)>1$ ).

We say that $G$ satisfies the strong vertex isoperimetric inequality if $h_{0}(G)>0$, and that $G$ satisfies the strong edge isoperimetric inequality if $h_{1}(G)>0$.

A graph $G$ has bounded degrees if there is an integer $M$ such that $\operatorname{deg}_{G}(v) \leq M$ for every $v \in V(G)$. Clearly,

Proposition 2.3. Let $G$ be a graph with bounded degrees. Then $h_{0}(G)>0$ if and only if $h_{1}(G)>0$. 


\section{Discharging IN INFINITE PLANAR GRAPHS}

Let $G$ be a locally finite plane graph. Let $F(G)$ be the set of faces of $G$. If $H$ is a finite subgraph of $G$, consider its induced embedding in the plane. Let $n_{H}, e_{H}$, and $f_{H}$ denote the number of vertices, edges, and faces of $H$, respectively. For $i \geq 0$, let $n_{i}$ be the number of vertices of degree $i$ in $H$, and let $f_{i}$ be the number of faces of $H$ of length $i$. Observe that $n_{H}=\sum_{i} n_{i}, 2 e_{H}=\sum_{i} i n_{i}=\sum_{i} i f_{i}$, and $f_{H}=\sum_{i} f_{i}$. Let $\alpha \in \mathbb{R}$ be a real number $(0 \leq \alpha \leq 3)$, and let $\alpha^{\prime}=3-\alpha$. Euler's formula implies that

$$
\begin{aligned}
12 & \leq 6\left(n_{H}-e_{H}+f_{H}\right) \\
& =6 \sum_{i \geq 0} n_{i}-\alpha \sum_{i \geq 0} i n_{i}-\alpha^{\prime} \sum_{i \geq 0} i f_{i}+6 \sum_{i \geq 0} f_{i} \\
& =\sum_{i \geq 0}(6-\alpha i) n_{i}+\sum_{i \geq 0}\left(6-\alpha^{\prime} i\right) f_{i} \\
& =\sum_{v \in V(H)}\left(6-\alpha \operatorname{deg}_{H}(v)\right)+\sum_{f \in F(H)}\left(6-\alpha^{\prime} \operatorname{deg}_{H}(f)\right) .
\end{aligned}
$$

Next, assign to each vertex $v \in V(G)$ and each face $f \in F(G)$ a charge

$$
c(v)=\alpha \operatorname{deg}_{G}(v)-6 \quad \text { and } \quad c(f)=\alpha^{\prime} \operatorname{deg}_{G}(f)-6,
$$

respectively. (If $\operatorname{deg}(f)=\infty$, then we set $c(f)=\infty$.)

Similarly, let $c_{H}(v)=\alpha \operatorname{deg}_{H}(v)-6(v \in V(H))$ and $c_{H}(f)=\alpha^{\prime} \operatorname{deg}_{H}(f)-6$ $(f \in F(H))$ denote the corresponding charges of vertices and faces of $H$. Inequality (3.1) shows that the sum of all charges in $H$ is negative.

Suppose that there is a set $\mathcal{R}$ of discharging rules that defines a redistribution of charges in $G$ in the following way. The rules determine that each vertex and each face sends some of its charge to other vertices and faces. Let $\varphi(x, y) \geq 0$ be the charge that is sent from $x$ to $y(x, y \in V(G) \cup F(G))$. Let $c^{-}(x)=\sum_{y} \varphi(x, y)$ be the charge sent from $x$ to other vertices and faces, and let $c^{+}(x)=\sum_{y} \varphi(y, x)$ be the charge received at $x$ from other vertices and faces when applying the rules. The new value of the charge at $x$ is then equal to $c^{*}(x)=c(x)-c^{-}(x)+c^{+}(x)$ and is called the final charge at $x$.

Discharging rules not only determine the values $\varphi(x, y)$, but also determine one or more paths $P(x, y)$ along which the charge is sent from $x$ to $y$. If $P(x, y)$ contains more than one path, it is also determined which proportion of $\varphi(x, y)$ is sent from $x$ to $y$ along particular paths in $P(x, y)$. Each path in $P(x, y)$ is a path in $G$, except that its first (and last) edge is an auxiliary edge joining the "center" of a face with an incident vertex if $x$ (or $y$ ) is a face of $G$. The maximum length of paths in $P(x, y)$, taken over all pairs $x, y$ such that $\varphi(x, y) \neq 0$, is called the impact range of $\mathcal{R}$.

Let $e$ be an (oriented) edge of $G$, or an auxiliary edge joining the center of a face with an incident vertex. Let $\varphi(e)$ be the sum of all charges $\varphi(x, y)$ (or corresponding parts of these charges) for which a path in $P(x, y)$ uses the edge $e$ in the given direction. The value $\varphi_{0}=\sup \{\varphi(e)\}$ is called the flow value of $\mathcal{R}$.

Let $F \in F(G)$, and let $x$ be a vertex incident with $F$. Let $\varphi^{\prime}(F, x)$ denote the total charge sent from $F$ through $x$, i.e., $\varphi^{\prime}(F, x)=\varphi(e)$, where $e$ is the auxiliary edge joining $F$ and $x$. The discharging rules $\mathcal{R}$ are said to be smooth if there is a constant $c_{0}^{\prime}$ such that for every $F \in F(G)$ and every segment $S$ on the facial walk 
of $F$ the following condition holds:

$$
\varphi^{\prime}(F, V(S))=\sum_{x \in V(S)} \varphi^{\prime}(F, x) \leq \alpha^{\prime}|V(S)|+c_{0}^{\prime} .
$$

The condition of smoothness is needed when there are arbitrarily large or even infinite faces.

If $X$ and $Y$ are finite subsets of $V(G) \cup F(G)$, then $c(X)=\sum_{x \in X} c(X)$ and $\varphi(X, Y)=\sum\{\varphi(x, y) \mid x \in X, y \in Y\}$. The same notation is used for other functions defined on vertices (and faces) of $G$. For example, $\operatorname{deg}(X)=\sum_{x \in X} \operatorname{deg}(x)$.

Theorem 3.1. Let $G$ be a connected infinite planar graph and let $0<\alpha<3$ and $\varepsilon>0$ be real numbers. Let $c$ and $c^{*}$ be the charge and the final charge, respectively, with respect to a set of smooth discharging rules of impact range at most $r$ and with flow value $\varphi_{0}$. Suppose that there is a finite set $\mathcal{F} \subset F(G)$ of faces such that the final charge of every face in $F(G) \backslash \mathcal{F}$ is nonnegative, and that there is a finite vertex set $U \subset V(G)$ such that $c^{*}(v) \geq \varepsilon$ for every vertex $v \in V(G) \backslash U$. Then the following hold:

(a) $G$ has exponential growth.

(b) If $G$ has bounded degree, then $h_{0}(G)>0$.

(c) If $r \leq 1$, then $h_{1}(G)>0$.

Proof. Let $X$ be a finite set of vertices of $G$ and let $\bar{X}=X \cup N_{r}(X)$. Let $H=G(X)$ be the subgraph of $G$ induced on $X$. Let $\mathcal{F}_{0} \subseteq F(H) \cap F(G)$ be the set of faces of $H$ that are also faces of $G$. Let $\mathcal{F}_{1}^{\prime}=F(H) \backslash \mathcal{F}_{0}$, and let $\mathcal{F}_{1} \subseteq F(G)$ be the set of faces of $G$ that are not in $\mathcal{F}_{0}$ but are incident with an edge with its endvertices in $\bar{X}$. Let

$$
\nu=-\sum\left\{c^{*}(v) \mid v \in V(G), c^{*}(v)<0\right\}
$$

and

$$
\phi=-\sum\left\{c^{*}(f) \mid f \in F(G), c^{*}(f)<0\right\} .
$$

The assumptions of the theorem imply

$$
c^{*}(X) \geq \varepsilon(|X|-|U|)-\nu
$$

and

$$
c^{*}\left(\mathcal{F}_{0}\right) \geq-\phi
$$

Every face in $\mathcal{F}_{1}$ is incident with at least two edges in $\delta X \cup E\left(G\left(N_{r}(X)\right)\right.$ ) (possibly twice with the same edge), and every such edge is contained in at most two faces in $\mathcal{F}_{1}$. Therefore,

$$
\begin{aligned}
\left|\mathcal{F}_{1}^{\prime}\right| \leq\left|\mathcal{F}_{1}\right| & \leq|\delta X|+\left|E\left(G\left(N_{r}(X)\right)\right)\right| \\
& \leq|\delta X|+3\left|N_{r}(X)\right|
\end{aligned}
$$

In the second inequality we used a well-known corollary of Euler's formula that a finite planar graph on $n$ vertices contains less than $3 n$ edges. Next,

$$
\begin{aligned}
c_{H}(F(H)) & =c_{H}\left(\mathcal{F}_{0}\right)+c_{H}\left(\mathcal{F}_{1}^{\prime}\right) \\
& =c\left(\mathcal{F}_{0}\right)+\alpha^{\prime} \operatorname{deg}_{H}\left(\mathcal{F}_{1}^{\prime}\right)-6\left|\mathcal{F}_{1}^{\prime}\right| \\
& \geq c\left(\mathcal{F}_{0}\right)+\alpha^{\prime} \operatorname{deg}_{H}\left(\mathcal{F}_{1}^{\prime}\right)-6\left(|\delta X|+3\left|N_{r}(X)\right|\right) .
\end{aligned}
$$


Since $c_{H}(v)=c(v)-\alpha \operatorname{deg}_{G}(v)+\alpha \operatorname{deg}_{H}(v)(v \in X)$, we have

$$
c_{H}(X)=c(X)-\alpha \operatorname{deg}_{G}(X)+\alpha \operatorname{deg}_{H}(X)=c(X)-\alpha|\delta X| .
$$

We will need estimates on the charge increase in $\mathcal{F}_{0}$ and in $X$. Since the discharging rules have impact range $\leq r$, the faces in $\mathcal{F}_{0}$ and vertices in $X$ send (and receive) nonzero charge only to (from) $\bar{X} \cup \mathcal{F}_{0} \cup \mathcal{F}_{1}$. Therefore,

$$
\begin{aligned}
c^{+}\left(\mathcal{F}_{0}\right)-c^{-}\left(\mathcal{F}_{0}\right)= & \varphi\left(\mathcal{F}_{0}, \mathcal{F}_{0}\right)+\varphi\left(\bar{X}, \mathcal{F}_{0}\right)+\varphi\left(\mathcal{F}_{1}, \mathcal{F}_{0}\right) \\
& -\varphi\left(\mathcal{F}_{0}, \mathcal{F}_{0}\right)-\varphi\left(\mathcal{F}_{0}, \bar{X}\right)-\varphi\left(\mathcal{F}_{0}, \mathcal{F}_{1}\right) \\
\leq & \varphi\left(\mathcal{F}_{1}, \mathcal{F}_{0}\right)+\varphi\left(\bar{X}, \mathcal{F}_{0}\right)-\varphi\left(\mathcal{F}_{0}, X\right)
\end{aligned}
$$

and

$$
\begin{aligned}
c^{+}(X)-c^{-}(X)= & \varphi(X, X)+\varphi\left(\mathcal{F}_{0}, X\right)+\varphi\left(N_{r}(X) \cup \mathcal{F}_{1}, X\right) \\
& -\varphi(X, X)-\varphi\left(X, \mathcal{F}_{0}\right)-\varphi\left(X, N_{r}(X) \cup \mathcal{F}_{1}\right) \\
\leq & \varphi\left(\mathcal{F}_{0}, X\right)+\varphi\left(N_{r}(X) \cup \mathcal{F}_{1}, X\right)-\varphi\left(X, \mathcal{F}_{0}\right) .
\end{aligned}
$$

Smoothness of the discharging rules is needed in the following estimate. Let us observe that the charge sent from $N_{r}(X) \cup \mathcal{F}_{1}$ to $X \cup \mathcal{F}_{0}$ either flows through some edge in $\delta X$ or goes from a face $F \in \mathcal{F}_{1}$ through a vertex in $X$ incident with $F$. This implies

$$
\begin{aligned}
\varphi\left(N_{r}(X) \cup \mathcal{F}_{1}, X \cup \mathcal{F}_{0}\right) & \leq \varphi_{0}|\delta X|+\sum_{F \in \mathcal{F}_{1}} \sum_{x \in X \cap V(F)} \varphi^{\prime}(F, x) \\
& \leq \varphi_{0}|\delta X|+\alpha^{\prime} \operatorname{deg}_{H}\left(\mathcal{F}_{1}^{\prime}\right)+c_{0}^{\prime}|\delta X|,
\end{aligned}
$$

where $c_{0}^{\prime}$ is the constant from the definition of the smoothness of $\mathcal{R}$. In the last inequality we used the fact that the number of facial segments $S$ of faces in $\mathcal{F}_{1}$ which have all their vertices in $X$ is at most $|\delta X|$, and that their total length is smaller than $\operatorname{deg}_{H}\left(\mathcal{F}_{1}^{\prime}\right)$.

Starting with (3.1) and applying inequalities (3.2)-(3.9), we get

$$
\begin{aligned}
-12 \geq & c_{H}(X)+c_{H}(F(H)) \\
\geq & c(X)-\alpha|\delta X|+c\left(\mathcal{F}_{0}\right)-6\left(|\delta X|+3\left|N_{r}(X)\right|\right)+\alpha^{\prime} \operatorname{deg}_{H}\left(\mathcal{F}_{1}^{\prime}\right) \\
= & c^{*}(X)-c^{+}(X)+c^{-}(X)+c^{*}\left(\mathcal{F}_{0}\right)-c^{+}\left(\mathcal{F}_{0}\right)+c^{-}\left(\mathcal{F}_{0}\right) \\
& -(\alpha+6)|\delta X|-18\left|N_{r}(X)\right|+\alpha^{\prime} \operatorname{deg}_{H}\left(\mathcal{F}_{1}^{\prime}\right) \\
\geq & c^{*}(X)-\varphi\left(\mathcal{F}_{0}, X\right)-\varphi\left(N_{r}(X) \cup \mathcal{F}_{1}, X\right)+\varphi\left(X, \mathcal{F}_{0}\right) \\
& +c^{*}\left(\mathcal{F}_{0}\right)-\varphi\left(\mathcal{F}_{1}, \mathcal{F}_{0}\right)-\varphi\left(X, \mathcal{F}_{0}\right)+\varphi\left(\mathcal{F}_{0}, X\right) \\
& -(\alpha+6)|\delta X|-18\left|N_{r}(X)\right|+\alpha^{\prime} \operatorname{deg}_{H}\left(\mathcal{F}_{1}^{\prime}\right) \\
= & c^{*}(X)+c^{*}\left(\mathcal{F}_{0}\right)-\varphi\left(N_{r}(X) \cup \mathcal{F}_{1}, X \cup \mathcal{F}_{0}\right) \\
& -(\alpha+6)|\delta X|-18\left|N_{r}(X)\right|+\alpha^{\prime} \operatorname{deg}_{H}\left(\mathcal{F}_{1}^{\prime}\right) \\
\geq & \varepsilon|X|-\varepsilon|U|-\nu-\phi \\
& -18\left|N_{r}(X)\right|-\left(\varphi_{0}+c_{0}^{\prime}+\alpha+6\right)|\delta X|
\end{aligned}
$$

Suppose now that $X$ is large, say $|X| \geq \frac{2}{\varepsilon}(\varepsilon|U|+\nu+\phi)$. Then (3.10) implies

$$
|\delta X|+\left|N_{r}(X)\right| \geq \frac{\varepsilon}{2 \rho}|X|,
$$

where $\rho=\max \left\{18, \varphi_{0}+c_{0}^{\prime}+\alpha+6\right\}$.

If $r \leq 1$ then $\left|N_{r}(X)\right| \leq|\delta X|$. Now, (3.11) shows that $|\delta X| \geq \frac{\varepsilon}{4 \rho}|X|$. Since this holds for every vertex set $X$ which is large enough and since $G$ is connected, it 
follows that $h_{1}(G)>0$. This proves (c). By Propositions 2.2 and 2.3 this proves also (a) and (b) in the case when $r \leq 1$.

Suppose now that $r \geq 1$. Inequality (3.11) holds for every finite vertex set $X$ with at least $\frac{2}{\varepsilon}(\varepsilon|U|+\nu+\phi)$ elements. In particular, it applies to the set $X_{1}=X \cup N_{1}(X)$ :

$$
\left|\delta X_{1}\right|+\left|N_{r}\left(X_{1}\right)\right| \geq \frac{\varepsilon}{2 \rho}\left|X_{1}\right| .
$$

As in the proof of Proposition 2.2. we see that $\left|\delta X_{1}\right| \leq 2\left(\left|N_{1}(X)\right|+\left|N_{1}\left(X_{1}\right)\right|\right)=$ $2\left|N_{2}(X)\right| \leq 2\left|N_{r+1}(X)\right|$. Since $\left|N_{r}\left(X_{1}\right)\right| \leq\left|N_{r+1}(X)\right|$, (3.12) gives

$$
\left|N_{r+1}(X)\right| \geq \frac{\varepsilon}{6 \rho}|X| .
$$

Consequently,

$$
\tau(G) \geq\left(1+\frac{\varepsilon}{6 \rho}\right)^{1 /(r+1)}>1 .
$$

This completes the proof of (a).

Suppose now that $\operatorname{deg}_{G}(v) \leq M$ (where $M \geq 3$ ) for every $v \in V(G)$. Then

$$
\begin{aligned}
\left|N_{r+1}(X)\right| & \leq\left|N_{1}(X)\right| \cdot\left(1+(M-1)+(M-1)^{2}+\cdots+(M-1)^{r}\right) \\
& \leq M^{r+1}\left|N_{1}(X)\right| .
\end{aligned}
$$

Therefore, $\left|N_{1}(X)\right| \geq M^{-(r+1)} \frac{\varepsilon}{6 \rho}|X|$ (for every finite vertex set $X$ with sufficiently many vertices). Since $G$ is connected, it follows that $h_{0}(G)>0$.

Let us observe that the proof of Theorem 3.1 gives explicit positive lower bounds on the exponential growth rate $\tau(G)$ and on the isoperimetric constants $h_{0}(G)$ and $h_{1}(G)$.

If $r=0$ (i.e., no discharging rules are used), the inequalities in the proof of Theorem 3.1 can be strengthened as follows. We shall assume that $U=\emptyset$ and $\mathcal{F}=\emptyset$, so that $\nu=\phi=0$. First of all, (3.4) can be replaced by

$$
\left|\mathcal{F}_{1}^{\prime}\right| \leq|\delta X|
$$

and (3.5) by

$$
c_{H}(F(H)) \geq c\left(\mathcal{F}_{0}\right)+\left(3 \alpha^{\prime}-6\right)|\delta X| .
$$

Inequality (3.9) is void since $\varphi\left(N_{r}(X) \cup \mathcal{F}_{1}, X \cup \mathcal{F}_{0}\right)=0$. Then (3.10) reduces to

$$
-12 \geq \varepsilon|X|-\left(\alpha+6-3 \alpha^{\prime}\right)|\delta X|=\varepsilon|X|-\left(9-4 \alpha^{\prime}\right)|\delta X| .
$$

Assuming that $\alpha^{\prime}<\frac{9}{4}$, this inequality implies

$$
h_{1}(G) \geq \frac{\varepsilon}{9-4 \alpha^{\prime}} .
$$

\section{Planar graphs of minimum Degree 6}

Let $T_{0}=\left[6^{3}\right]$ be the tessellation of the plane with equilateral triangles (see Figure 1). We may assume that the origin of $\mathbb{R}^{2}$ is a vertex of $T_{0}$. Denote that vertex by $z_{0}$. Let $z$ be a vertex of $T_{0}$ distinct from $z_{0}$. Let $\Gamma \leq \operatorname{Aut}\left(T_{0}\right)$ be the infinite cyclic group generated by the translation of $\mathbb{R}^{2}$ which maps $z_{0}$ to $z$. Then $T_{0}^{z}=T_{0} / \Gamma$ is a tessellation of the "flat" cylinder by equilateral triangles. (The graph of $T_{0}^{z}$ may have loops or multiple edges if $\operatorname{dist}_{T_{0}}\left(z, z_{0}\right) \leq 2$.) If $r$ is an integer, denote by $T_{0}(r)$ and $T_{0}^{z}(r)$ the submap of $T_{0}$ or $T_{0}^{z}$, respectively, consisting of all vertices and faces that are at distance $\leq r$ from $z_{0}$. 


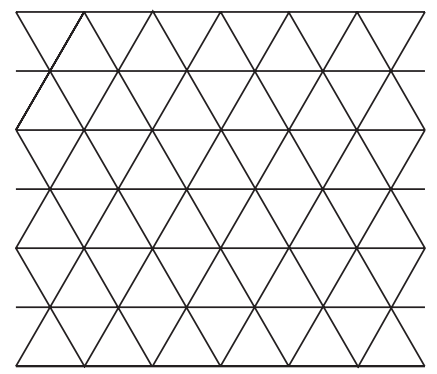

Figure 1. A fragment of the tessellation $\left[6^{3}\right]$

Let $G_{0}$ and $G$ be plane graphs and let $H_{0}$ be a subgraph of $G_{0}$. Suppose that $G$ contains a subgraph $H$ isomorphic to $H_{0}$ such that a closed walk in $H$ bounds a face of $G$ if and only if the corresponding closed walk in $H_{0}$ bounds a face of $G_{0}$. Then we say that $G$ contains $H_{0}$ (as a submap).

Theorem 4.1. Let $G$ be a finite planar graph, $r$ a positive integer, and let $m \geq$ $(3 r+1)^{2}$. Suppose that $v$ is a vertex of $G$ such that all vertices at distance $\leq m$ from $v$ are of degree 6 and are incident only with triangular faces. Then one of the following holds:

(a) $G$ contains $T_{0}(r)$ as a submap.

(b) $G$ contains $T_{0}^{z}(3 r)$ as a submap, where $\operatorname{dist}_{T_{0}}\left(z, z_{0}\right) \leq 3 r-1$.

Proof. Let $U \subseteq V(G)$ be the set of vertices of $G$ at distance at most $m$ from $v$. Fix a triangle $A_{0}$ incident with $v$. Let $C$ be a cycle of $G$ with $V(C) \subseteq U$. Denote by $\operatorname{int}(C)$ the component of $\mathbb{R}^{2} \backslash C$ which does not contain $A_{0}$. For $u \in V(C)$, let $\delta(u, C)$ be the number of edges incident with $u$ which are embedded in int $(C)$, and let $\eta(C)=\sum_{u \in V(C)}(\delta(u, C)-2)$.

The following property of $\eta(C)$ is easy to prove:

(P1) If $P$ is a path in $\operatorname{int}(C)$ with $V(P) \subseteq U$ such that $P \cap C$ are the endvertices of $P$, let $C_{1}$ and $C_{2}$ be the two cycles of $C \cup P$ distinct from $C$. Then

$$
\eta\left(C_{1}\right)+\eta\left(C_{2}\right)=\eta(C)-6 .
$$

If $C_{2}$ in (P1) is a facial triangle, then (P1) implies that $\eta\left(C_{1}\right)=\eta(C)$. Repetitive use of this property shows that

(P2) If a cycle $C_{1}$ with $V\left(C_{1}\right) \subseteq U$ is obtained from $C$ by consecutively adding facial triangles with at least one edge in common with the current cycle, then $\eta\left(C_{1}\right)=\eta(C)$.

Properties (P1) and (P2) can be used to prove

(P3) Suppose that $|V(C)|=k$ and that all vertices in $\operatorname{int}(C)$ that are at distance at most $k-2$ from $C$ are in $U$. If $\eta(C)<0$, then $\eta(C)=-6$ and all vertices in $\operatorname{int}(C)$ are at distance at most $k-3$ from $C$.

The proof of (P3) is by induction on $k$. Suppose first that $k=3$. Since $\eta(C)<0$, there is a vertex $u \in V(C)$ with $\delta(u, C) \leq 1$. It is easy to see that $\delta(u, C)=1$ is not possible. Therefore, $\delta(u, C)=0$. This implies that $C$ is a facial triangle, and hence (P3) holds.

Suppose now that $k \geq 4$. Let $A$ be the set of facial triangles in $\operatorname{int}(C)$ that have a vertex in $V(C)$. Let $E^{\prime}$ be the set of edges in $\operatorname{int}(C)$ that are contained in 
precisely one triangle in $A$. If $E^{\prime}=\emptyset$ then $A$ partitions int $(C)$ into triangles, and (P2) implies that $\eta(C)$ is equal to $\eta(T)=-6$ for some $T \in A$. Hence, we may assume that $E^{\prime} \neq \emptyset$. Then $E^{\prime}$ can be partitioned into cycles $C_{1}, \ldots, C_{r}(r \geq 1)$ with disjoint interiors. The cycles $C_{i}$ are boundaries of faces of $C \cup A \operatorname{in} \operatorname{int}(C)$ that are not triangles in $A$. Since $C_{1}, \ldots, C_{r}$ can be obtained from $C$ by successively applying operations in (P1) and (P2),

$$
\eta\left(C_{1}\right)+\cdots+\eta\left(C_{r}\right)=\eta(C)-6(r-1) .
$$

A simple calculation shows that $\left|E^{\prime}\right| \leq k+\eta(C)<k$. This implies that $\left|V\left(C_{i}\right)\right|<k$ for $i=1, \ldots, r$. By the induction hypothesis, either $\eta\left(C_{i}\right)=-6$ or $\eta\left(C_{i}\right) \geq 0$. Since $\eta(C)<0$, (4.1) implies that $\eta\left(C_{i}\right)=-6$ for every $i$, and, consequently, $\eta(C)=-6$. Moreover, all vertices in $\operatorname{int}\left(C_{i}\right)$ are at distance at most $k-4$ from $C_{i}$, $i=1, \ldots, r$. Therefore, all vertices in $\operatorname{int}(C)$ are at distance at most $k-3$ from $C$. This completes the proof of (P3).

Now we are prepared to prove the theorem. Let $q$ be the largest integer $\leq m$ such that the $q$-ball $B_{q}(G, v)$ is isomorphic to the $q$-ball $B_{q}\left(T_{0}, z_{0}\right)$ in $T_{0}$. We are done if $q \geq r$, so suppose that $1 \leq q \leq r-1$.

Denote by $C$ the outer cycle of $B_{q}(G, v)$. Note that $|V(C)|=6 q$ and that $\eta(C)=6$. Since $B_{q+1}(G, v)$ is not isomorphic to $B_{q+1}\left(T_{0}, z_{0}\right)$, there are distinct vertices $u_{1}, u_{2} \in V(C)$ that are not adjacent on $C$, and there are edges $e_{1}, e_{2}$ incident with $u_{1}$ and $u_{2}$, respectively, such that either $e_{1}=e_{2}$, or $e_{1}$ and $e_{2}$ have a vertex $u$ in $\operatorname{int}(C)$ in common. Let $C_{1}$ and $C_{2}$ be the cycles in $C+e_{1}+e_{2}$ distinct from $C$. For $i=1,2,\left|V\left(C_{i}\right)\right| \leq 6 q$ and all vertices at distance at most $6 q-2$ from $C_{i}$ are in $U$. By $(\mathrm{P} 2), \eta\left(C_{1}\right)+\eta\left(C_{2}\right)=\eta(C)-6=0$. If $\eta\left(C_{i}\right)<0$, then by (P3), $\eta\left(C_{i}\right)=-6$. This implies that $\delta\left(u_{1}, C_{i}\right)=\delta\left(u_{2}, C_{i}\right)=\delta\left(u, C_{i}\right)=0$, since all vertices $u^{\prime}$ on $C \cap C_{i}$ distinct from $u_{1}$ and $u_{2}$ have $\delta\left(u^{\prime}, C_{i}\right)=\delta\left(u^{\prime}, C\right) \geq 2$. Clearly, this is not possible. Consequently, $\eta\left(C_{1}\right)=\eta\left(C_{2}\right)=0$.

Let $R$ be a shortest cycle in $G$ with the following properties:

(a) All vertices of $R$ are at distance at most $(3 r+1)(3 r-|V(R)|)$ from $v$. (In particular, $V(R) \subseteq U$ and $|V(R)| \leq 3 r-1$.)

(b) $\eta(R)=0$.

Since $q \leq r-1,|V(C)|=6 q \leq 6 r-6$. Assuming $\left|V\left(C_{1}\right)\right| \leq\left|V\left(C_{2}\right)\right|$, we have $\left|V\left(C_{1}\right)\right| \leq \frac{1}{2}|V(C)|+2 \leq 3 r-1$. Since all vertices on $C_{1}$ are at distance at most $q+1$ from $v, C_{1}$ satisfies (a) and (b). Therefore, $R$ exists.

Suppose that there exists a path $P$ in $G$ such that $P$ intersects $R$ precisely at its endvertices $u_{1}, u_{2}$, and such that the length of $P$ is smaller than the distance from $u_{1}$ to $u_{2}$ on $R$. Consider the cycles $R_{1}, R_{2} \subset R \cup P$ distinct from $R$. (P1) and (P3) imply that $\eta\left(R_{1}\right)=0$ and $\eta\left(R_{2}\right)=-6$ (or vice versa). Since $R_{1}$ is shorter than $R$ and every vertex on $R_{1}$ is at distance at most $3 r(3 r-k)+\frac{k}{2}$ from $v, R_{1}$ satisfies (a)-(b) and contradicts the minimality of $R$. This shows that $R$ is an isometric subgraph of $G$.

Fix any vertex $v_{0}$ on $R$. Then $R$ determines a path $P(R)$ in $T_{0}$ from $z_{0}$ to some vertex $z \in V\left(T_{0}\right)$ such that $v_{0}$ corresponds to the ends of the path and such that at each other vertex on $P(R)$, the number of edges on the "left" side of the path is equal to $\delta(u, R)$, where $u$ is the corresponding vertex on $R$. Since $R$ is an isometric subgraph in $G$, the path $P(R)$ is also isometric in $T_{0}$. The path $P(R)$ can be extended to a two-way-infinite path $P_{\infty}(R)$ in $T_{0}$. Since $\eta(R)=0$, it is easy to see that $P_{\infty}(R)$ does not cross itself and that it gives rise to a cycle $R^{\prime}$ in $T_{0}^{z}$. 
We claim that the vertices of $G$ at distance at most $3 r$ from $R$ form a submap of $G$ isomorphic to the distance-3r neighborhood of $R^{\prime}$ in $T_{0}^{z}$. If not, then we proceed in the same way as above when we proved existence of $C_{1}$ and $C_{2}$. Here, we obtain cycles $R_{1}$ and $R_{2}$ in $G$ whose length is not larger than the length $k$ and such that $\eta\left(R_{1}\right)+\eta\left(R_{2}\right)=\eta(R)-6=-6$. Then $\eta\left(R_{1}\right)=0$ and $\eta\left(R_{2}\right)=-6$ (or vice versa). If the length of $R_{1}$ is smaller than $k$, we get a contradiction to the minimality of $R$. Otherwise, the length of $R_{2}$ is either 3 or 4 . Since $R_{2}$ is not a facial triangle and since $\eta\left(R_{2}\right)=-6$, we easily get a contradiction. This completes the proof.

If $G$ is a graph, its minimum degree is denoted by $\delta(G)$. We also define its minimum essential degree $\delta_{\text {ess }}(G)$ as the minimum integer $d$ such that there are infinitely many vertices of degree $d$. If such $d$ does not exist, then $\delta_{\text {ess }}(G)=\infty$.

A simple application of Theorem 3.1(c) (with $\alpha=1$ and $r=0$, i.e., no discharging rules at all) shows that $h_{1}(G)>0$ if $\delta_{\text {ess }}(G) \geq 7$. This result was proved by Dodziuk [6]. Here we show that a similar result holds when $\delta_{\text {ess }}(G)=6$.

Theorem 4.2. Let $G$ be a connected infinite planar graph with $\delta_{\mathrm{ess}}(G) \geq 6$. If $G$ has subexponential growth, then either $G$ contains every finite submap of $T_{0}$, or there exists a vertex $z \in V\left(T_{0}\right)$ such that $G$ contains every finite submap of $T_{0}^{z}$.

Proof. Let $U$ be the set of vertices whose degree is at most 5 , and let $U_{6}$ be the set of vertices of degree 6 . Let $m$ be an arbitrary positive integer. We shall apply the discharging method with $\alpha=1$ and use the following discharging rule.

Let $x$ be a face of length $\geq 4$ or a vertex which is not in $U_{6}$. Let $P(x)$ be the set of all paths of length at most $m+1$ that start at $x$ and whose other vertices are all in $U_{6}$. (If $x$ is a face, then the first edge of every such path is an auxiliary edge joining the "center" of $x$ with an incident vertex of degree 6 in $G$.) There are at most $\operatorname{deg}_{G}(x) \cdot 6^{m+1}$ such paths, and the discharging rule determines that $x$ sends charge $\varepsilon=\left(2 \operatorname{deg}_{G}(x) \cdot 6^{m+1}\right)^{-1}$ along every path in $P(x)$ to the terminal vertex of the path. Clearly, $c^{*}(x) \geq c(x)-\frac{1}{2} \geq \frac{1}{2}$ if $x \in V(G) \backslash\left(U \cup U_{6}\right)$. Also, every face has nonnegative final charge, and every vertex that belongs to a path in some $P(x)$ has its final charge $\geq \varepsilon$.

Theorem 3.1 can be applied to the given discharging process, showing that either $G$ has exponential growth, or there is a vertex $v \in U_{6}$ that has not received any charge. Since $G$ has subexponential growth, $v$ exists, and every vertex at distance at most $m$ from $v$ is of degree 6 and incident only with triangular faces.

As $m$ is arbitrarily large, Theorem 4.1 implies that there is an infinite sequence of pairs $\left(r_{i}, z_{i}\right) \in \mathbb{N} \times V\left(T_{0}\right)$, where $r_{i} \rightarrow \infty$ as $i \rightarrow \infty$ and $\operatorname{dist}_{T_{0}}\left(z_{i}, z_{0}\right) \leq 3 r_{i}-1$, such that $G$ contains either $T_{0}\left(r_{i}\right)$ or $T_{0}^{z_{i}}\left(3 r_{i}\right)$. If the first case occurs infinitely often or if limsup $\operatorname{dist}_{T_{0}}\left(z_{i}, z_{0}\right)=\infty$, then $G$ contains every finite submap of $T_{0}$. Otherwise, there exists $z \in V\left(T_{0}\right)$ such that $z_{i}=z$ for infinitely many values of $i$. In that case, $G$ contains every finite submap of $T_{0}^{z}$. This completes the proof.

\section{KOTZIG'S THEOREM FOR INFINITE GRAPHS}

Let $u v \in E(G)$. The sum of degrees, $\operatorname{deg}(u)+\operatorname{deg}(v)$, of the ends of $u v$ is called the weight of the edge $u v$. If $\operatorname{deg}(u) \leq \operatorname{deg}(v)$, then the edge $u v$ is said to be of type $(\operatorname{deg}(u), \operatorname{deg}(v))$. The edge is very light if its weight is at most 11 or if it is of type $(3,9)$ or $(3,10)$. The edge is light if it is either very light or of one of the following types: $(3,11),(3,12),(4,8)$, or $(6,6)$. 
Kotzig [15] proved that every finite 3 -connected planar graph contains a very light edge. Borodin [4] showed that the same result holds under the weaker hypothesis that the minimum degree is $\geq 3$. Grünbaum and Shephard [11] and Stehling [21] extended Kotzig's theorem to graphs of doubly periodic tilings and of normal tilings of the plane, respectively, by showing that they always contain light edges. Let us observe that the graphs of normal tilings have quadratic growth.

The main result of this section is an extension of Grünbaum and Shephard's result to arbitrary planar graphs that do not satisfy the strong isoperimetric inequality. In particular, this holds for all graphs of subexponential growth.

Theorem 5.1. Let $G$ be a connected infinite planar graph with $\delta_{\mathrm{ess}}(G) \geq 3$. If $G$ has only finitely many light edges, then $h_{1}(G)>0$.

Proof. Suppose that $G$ is a connected infinite planar graph with $l<\infty$ light edges. We define the charge for vertices and faces of $G$ as in Section 3 with $\alpha=1$. Then all faces have nonnegative charge and all vertices of degree $\geq 7$ have positive charge $c(v)=\operatorname{deg}(v)-6$. Let $\phi(3)=\frac{21}{20}, \phi(4)=\frac{3}{5}, \phi(5)=\frac{1}{4}$, and $\phi(6)=\frac{1}{20}$. We shall apply the following set of discharging rules.

Rule 1: Suppose that $f$ is a face with $\operatorname{deg}(f) \geq 4$, and that $v$ is a vertex incident with $f$. If $4 \leq \operatorname{deg}(v) \leq 6$, then $\varphi(f, v)=\phi(\operatorname{deg}(v))$. If $\operatorname{deg}(v)=3$, then $\varphi(f, v)=1$. Otherwise, $\varphi(f, v)=0$.

Rule 2: Suppose that $u v \in E(G)$ is an edge of $G$, with $\operatorname{deg}(u) \geq 7$ and $\operatorname{deg}(v) \leq$ 6. If both faces containing $u v$ are triangles, then $\varphi(u, v)=\phi(\operatorname{deg}(v))$. If only one face containing $u v$ is a triangle, then $\varphi(u, v)=\frac{1}{2} \phi(\operatorname{deg}(v))$. If $u v$ is not contained in a triangular face and $\operatorname{deg}(v)=3$, then $\varphi(u, v)=\frac{1}{20}$. Otherwise, $\varphi(u, v)=0$.

Rules 1 and 2 clearly satisfy the premises of Theorem 3.1 with impact range $r=1$ and with flow value $\varphi_{0}=\frac{21}{20}$. Therefore, it suffices to see that the final charge is nonnegative for all faces that are not incident with light edges, and that the final charge is $\geq \varepsilon=\frac{1}{10}$ for all vertices of degree $\geq 3$ that are not incident with a light edge.

Let $f \in F(G)$. If $\operatorname{deg}(f)=3$, then $c^{*}(f)=c(f)=0$. Otherwise, suppose that $\operatorname{deg}(f) \geq 4$ and that $f$ is not incident with a light edge. Clearly, for any two consecutive vertices $u, v$ on the facial walk of $f$,

$$
\varphi(f, u)+\varphi(f, v) \leq 1 .
$$

This implies that $c^{-}(f) \leq \frac{1}{2} \operatorname{deg}(f)$, and hence $c^{*}(f)=c(f)-c^{-}(f) \geq \frac{3}{2} \operatorname{deg}(f)-$ $6 \geq 0$.

Suppose now that $v$ is a vertex of degree $d \geq 3$ that is not incident with a light edge. If $d=3$, then it is easy to see that $c^{+}(v) \geq 3+\frac{1}{10}$, and hence $c^{*}(v) \geq \frac{1}{10}$. If $4 \leq d \leq 6$, then $c^{+}(v)=d \cdot \phi(d)$. Therefore, $c^{*}(v)=\frac{2}{5}$ if $d=4, c^{*}(v)=\frac{1}{4}$ if $d=5$, and $c^{*}(v)=\frac{3}{10}$ if $d=6$.

If $7 \leq d \leq 8$, then $v$ sends charge only to vertices of degrees 5 and 6 . To any two consecutive neighbors $u, w$ (with respect to the clockwise orientation around $v$ ), $v$ sends at most charge $\varphi(5)=\frac{1}{4}$. Therefore, $c^{*}(v) \geq d-6-\frac{d}{2} \cdot \frac{1}{4} \geq \frac{1}{8}$.

If $9 \leq d \leq 12$, then $v$ sends charge only to vertices of degrees 4,5 , and 6 . To any two consecutive neighbors it sends at most $\varphi(4)=\frac{3}{5}$. Therefore, $c^{*}(v) \geq$ $d-6-\frac{d}{2} \cdot \frac{3}{5} \geq \frac{3}{10}$.

If $d \geq 13$, then $v$ sends at most $\varphi(3)$ to any two consecutive neighbors. Thus, $c^{*}(v) \geq d-6-\frac{d}{2} \cdot \frac{21}{20} \geq \frac{7}{40}$. This completes the proof. 

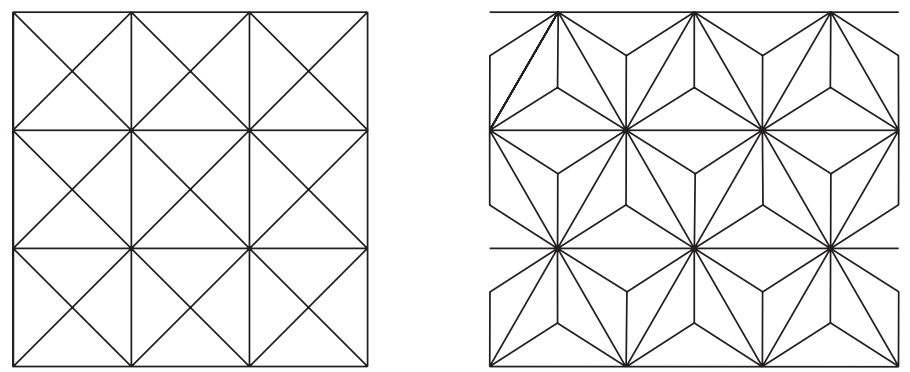

Figure 2. Fragments of tessellations $\left[4.8^{2}\right]$ and $\left[3.12^{2}\right]$

Theorem $\left[5.1\right.$ is best possible, as shown by the following examples. Let $T_{0}=\left[6^{3}\right]$ be the tessellation of the plane by equilateral triangles, let $T_{1}=\left[4.8^{2}\right]$ be the triangulation of the plane obtained from the tessellation with squares by adding a vertex of degree 4 in each square, and let $T_{2}=\left[3.12^{2}\right]$ be the triangulation of the plane obtained from $\left[6^{3}\right]$ by inserting a vertex of degree 3 into each triangle of $\left[6^{3}\right]$. See Figure 2. These examples contain only edges of types $(6,6),(4,8)$, and $(3,12)$, respectively. We also define the cylindrical quotients $T_{1}^{z}$ and $T_{2}^{z}$ in the same way as $T_{0}^{z}$ (cf. Section 4).

We need some further notation. Let $T=T_{0}$ or $T=T_{0}^{z}$, and let $\mathcal{F}$ be a set (possibly infinite) of facial triangles of $T$ such that every vertex of $T$ is incident with at most one triangle in $\mathcal{F}$. Next, add into each face of $T$ which is not in $\mathcal{F}$ a new vertex of degree 3 joined to the vertices on the boundary of that face. Let $T(\mathcal{F})$ be the resulting map. Note that $T_{0}(\emptyset)=T_{2}$ and $T_{0}^{z}(\emptyset)=T_{2}^{z}$.

Theorem 5.2. Let $G$ be a connected infinite planar graph of subexponential growth and with $\delta_{\mathrm{ess}}(G) \geq 3$.

(a) If $G$ has only finitely many very light edges and finitely many edges of types $(4,8),(6,6)$, and $(3,11)$, then either $G$ contains every finite submap of $T_{2}$, or $G$ contains every finite submap of some $T_{2}^{z}$.

(b) If $G$ has only finitely many very light edges and finitely many edges of types $(4,8)$ and $(6,6)$, then $G$ contains arbitrarily large finite submaps of some map of the form $T_{0}(\mathcal{F})$ or $T_{0}^{z}(\mathcal{F})$.

(c) If $G$ has only finitely many very light edges, then either $G$ contains arbitrarily large finite submaps of some map of the form $T_{0}(\mathcal{F})$ or $T_{0}^{z}(\mathcal{F})$, or $G$ contains every finite submap of a map of the form $T_{0}, T_{0}^{z}, T_{1}$, or $T_{1}^{z}$.

Proof. The proof is a continuation of the proof of Theorem 5.1 by adding similar discharging rules as used in the proof of Theorem 4.2, with an application of Theorem 4.1 and its extension from $T_{0}$ to $T_{1}$ and $T_{2}$. We leave the details to the reader. Let us only observe that in case (b), one cannot guarantee that $G$ contains every finite submap of $T_{0}(\mathcal{F})$ or of some $T_{0}^{z}(\mathcal{F})$. The reason that this is possible in the other cases is that $T_{0}, T_{1}, T_{2}$ and their quotients have automorphism groups with only finitely many orbits.

The following results are proved analogously:

Corollary 5.3. Let $G$ be a connected infinite planar graph of minimum essential degree $\delta_{\mathrm{ess}}(G) \geq 4$ and with $h_{1}(G)=0$. Then $G$ has infinitely many edges of weight 
at most 12. If $G$ has subexponential growth and does not have infinitely many edges of weight at most 11 , then there is a map $T$ isomorphic to $T_{0}, T_{0}^{z}, T_{1}$, or $T_{1}^{z}$ such that $G$ contains every finite submap of $T$.

Corollary 5.4. Let $G$ be a connected infinite planar graph of minimum essential degree $\delta_{\mathrm{ess}}(G) \geq 5$ and with subexponential growth. If $G$ does not have infinitely many edges of weight at most 11 , then either $G$ contains every finite submap of $T_{0}$, or $G$ contains every finite submap of some $T_{0}^{z}$.

It is easy to construct examples which show that Theorem 5.2 and Corollaries 5.3 and 5.4 cannot be improved to include infinite submaps of $\left[6^{3}\right],\left[4.8^{2}\right]$, or $\left[3.12^{2}\right]$ (or their cylindrical quotients).

The following corollary of Theorem 5.2(b) is an extension of Grünbaum and Shephard's result 11] which states that a doubly periodic tiling of the plane has an edge of weight at most 14 except when it is isomorphic to $\left[3.12^{2}\right]$. Our result below extends Grünbaum and Shephard's result to doubly periodic tilings without edges of weight at most 13. Such tilings are obtained as follows. Let $Q$ be a 6 -regular triangulation of the torus. (Such graphs have a simple three-parameter description. See, e.g., [1.) Select a set $\mathcal{F}$ of facial triangles of $Q$ such that every vertex of $Q$ is incident with at most one triangle in $\mathcal{F}$. Then, add into each triangular face which is not in $\mathcal{F}$ a new vertex of degree 3 joined to the vertices on the boundary of that face. Let $Q(\mathcal{F})$ be the resulting toroidal map. Then a doubly periodic tiling of the plane with minimum weight 14 ( or 15 if $\mathcal{F}=\emptyset$ ) is obtained as the universal cover of $Q(\mathcal{F})$. Note that the tiling is isomorphic to $\left[3.12^{2}\right]$ if $\mathcal{F}=\emptyset$.

Corollary 5.5. A doubly periodic tiling of the plane has no edges of weight at most 13 if and only if it is a universal cover of some toroidal triangulation $Q(\mathcal{F})$ described above.

The proof is left to the reader.

\section{Hyperbolic tessellations}

Let $G$ be a planar graph. Then its minimum face length is denoted by $\delta^{*}(G)$. We also define its minimum essential face length $\delta_{\mathrm{ess}}^{*}(G)$ as the minimum integer $d$ such that there are infinitely many faces of length $d$. If such $d$ does not exist, then $\delta_{\text {ess }}^{*}(G)=\infty$.

If $G$ is an infinite planar graph with $\delta_{\text {ess }}(G) \geq p$ and $\delta_{\text {ess }}^{*}(G) \geq q$, where $\frac{1}{p}+\frac{1}{q}<\frac{1}{2}$, then we say that $G$ is strongly hyperbolic. (This is a special case of more general hyperbolic maps as introduced, for example, by Woess [22].) Let $\alpha=3-\frac{6}{q}, \alpha^{\prime}=\frac{6}{q}$, and let $\kappa=\left(\frac{1}{p}+\frac{1}{q}\right)-\frac{1}{2}<0$. The value of $\kappa$ can be viewed as an upper bound on the (negative) curvature, see, e.g., 3]. Then for all vertices $v \in V(G)$ of degree at least $p$

$$
c(v)=\alpha \operatorname{deg}(v)-6 \geq \alpha p-6=-6 p \kappa>0,
$$

and for all faces $f \in F(G)$ of length at least $q$

$$
c(f)=\alpha^{\prime} \operatorname{deg}(f)-6 \geq \alpha^{\prime} q-6=0 .
$$

If $\delta(G) \geq p$ and $\delta^{*}(G) \geq q$, then inequality (3.17) in the remark after Theorem 3.1 shows that

$$
h_{1}(G) \geq \frac{2 p q|\kappa|}{3 q-8} .
$$


This bound compares well with the result of Baues and Peyerimhoff [3], which is just slightly better than (6.1).

More generally, the above method can be used to get explicit positive lower bounds on $h_{1}(G)$ in more general hyperbolic cases introduced by Woess [22]. We have to remark, though, that our results are more general than those in [3, 22, since we do not require tessellations to be normal or to have only one end.

\section{LIGHT SUBGRAPHS}

Kotzig's theorem (cf. Section 5) motivated further research on finite 3-connected planar graphs. Fabrici and Jendrol' 7 proved that for every integer $k$, every finite 3 -connected planar graph $G$ that contains at least one $k$-path, also contains a $k$ path whose vertices all have degree at most $5 k$ in $G$. Therefore, the $k$-path $P_{k}$ is said to be light in the set of 3-connected planar graphs. On the other hand, no other connected planar graph is light 7].

We extend these results to infinite graphs. Let us observe that the assumption on 3-connectivity is necessary already for the path $P_{3}$ on three vertices (while for $P_{2}$ the weaker condition $\delta(G) \geq 3$ suffices, as shown by Theorem 5.1).

Theorem 7.1. Let $k \geq 1$ be an integer and let $G$ be a 3-connected infinite planar graph of subexponential growth. Then $G$ contains infinitely many (disjoint) $k$-paths whose vertices have degree at most $6 k$.

Proof. A vertex of $G$ of degree at most $6 k$ is called a minor vertex. Other vertices are major. Let $M(G)$ denote the set of major vertices of $G$.

Let $\ldots v_{1} v_{2} \ldots v_{n} \ldots$ be a facial walk of a face $F$, and $i, j$ integers such that $i+2 \leq j \leq i+k, v_{i}$ and $v_{j}$ are major vertices and $v_{i+1}, v_{i+2}, \ldots, v_{j-1}$ are minor vertices. Then we say that $v_{i}$ and $v_{j}$ are joinable in $F$. Let $G^{\prime}$ be the graph that is obtained from $G$ by adding all edges between all pairs of vertices that are joinable in some face. Since $G$ is 3-connected, $G^{\prime}$ is a planar graph (without multiple edges). Clearly, $G^{\prime}$ has subexponential growth and $M\left(G^{\prime}\right)=M(G)$. Therefore, it suffices to prove that $G^{\prime}$ has infinitely many $k$-paths whose vertices are not in $M\left(G^{\prime}\right)$.

Let $G^{\prime \prime}=G^{\prime}\left(M\left(G^{\prime}\right)\right)$ be the induced subgraph of $G^{\prime}$ on major vertices. We may assume that $M\left(G^{\prime}\right)$ is infinite. Since $G^{\prime \prime}$ has subexponential growth, Theorem 4.2 implies that $G^{\prime \prime}$ has infinitely many vertices of degree at most 6 .

Let $v$ be any major vertex of $G^{\prime}$ whose degree in $G^{\prime \prime}$ is at most 6 . Since $G$ and hence also $G^{\prime}$ is 3-connected, the link of $v$ (which consists of all vertices and edges that are on a common face with $v$ but disjoint from $v$ ) is a cycle in $G^{\prime}$. Since $\operatorname{deg}_{G^{\prime}}(v) \geq 6 k+1$ and $\operatorname{deg}_{G^{\prime \prime}}(v) \leq 6$, there is a sequence of $k$ consecutive neighbors of $v$ (with respect to the clockwise ordering around $v$ in the plane), none of which is a major vertex. By the definition of $G^{\prime}$ it follows that the corresponding segment on the link of $v$ contains no major vertices. Therefore, this segment contains a $k$-path $P(v)$.

This proves that there is an infinite set of $k$-paths $P(v)$ that contain only minor vertices, and each $P(v)$ contains a neighbor of $v$. Every such $k$-path corresponds to only finitely many distinct vertices $v$. This easily implies that there is an infinite subset of these paths whose members are distinct and pairwise disjoint. 
Let $P=v_{1} v_{2} v_{3} \ldots$ be a one-way-infinite path in $G$. We say that $P$ has average degree $\leq D$ if, for every positive integer $k$,

$$
\sum_{i=1}^{k} \operatorname{deg}_{G}\left(v_{i}\right) \leq k D
$$

Not every planar graph of polynomial growth contains an infinite path with bounded average degree. We have found a complicated example in which for every infinite path $P=v_{1} v_{2} v_{3} \ldots$ and every $k \geq 1$

$$
\sum_{i=1}^{k} \operatorname{deg}_{G}\left(v_{i}\right)=\Theta(k \log k) .
$$

We propose the following:

Conjecture 7.2. There is a constant $C$ such that every connected infinite planar graph with subexponential growth contains a one-way-infinite path $P=v_{1} v_{2} v_{3} \ldots$ such that for every $k \geq 1$

$$
\sum_{i=1}^{k} \operatorname{deg}_{G}\left(v_{i}\right) \leq C k \log k .
$$

A proof of Conjecture 7.2 would solve an open problem about finite graphs (cf., e.g., [7, Problem 3]).

\section{REFERENCES}

[1] A. Altshuler, Hamiltonian circuits in some maps on the torus, Discrete Math. 1 (1972) 299314. MR 45:6651

[2] K. Appel, W. Haken, Every planar map is four colorable. Part I: Discharging, Ill. J. Math. 21 (1977) 429-490. MR 58:27598a

[3] O. Baues, N. Peyerimhoff, Curvature and geometry of tessellating plane graphs, Discrete Comput. Geom. 25 (2001) 141-159. MR 2001k:57004

[4] O. V. Borodin, A generalization of Kotzig's theorem and prescribed edge coloring of planar graphs, Mat. Zametki 48 (1990) no. 6, 22-28; English transl., Math. Notes 48 (1990), 11861190. MR 92e:05046

[5] A. Calogero, Strong isoperimetric inequality for the edge graph of a tiling of the plane, Arch. Math. (Basel) 61 (1993) 584-595. MR 94m:52024

[6] J. Dodziuk, Difference equations, isoperimetric inequality and transience of certain random walks, Trans. Amer. Math. Soc. 284 (1984) 787-794. MR 85m:58185

[7] I. Fabrici, S. Jendrol', Subgraphs with restricted degrees of their vertices in planar 3-connected graphs, Graphs Combin. 13 (1997) 245-250. MR 98h:05112

[8] P. Gerl, Random walks on graphs with a strong isoperimetric inequality, J. Theoret. Probab. 1 (1988) 171-187. MR 89g:60216

[9] M. Gromov, Hyperbolic manifolds (according to Thurston and Jorgensen), Bourbaki Seminar, Vol. 1979/80, Lecture Notes in Math. 842, Springer, Berlin-New York, 1981, pp. 40-53. MR 84b:53046

[10] M. Gromov, Hyperbolic groups. Essays in group theory, Math. Sci. Res. Inst. Publ. 8, Springer, New York, 1987, pp. 75-263. MR 89e:20070

[11] B. Grünbaum, G. C. Shephard, Analogues for tilings of Kotzig's theorem on minimal weights of edges, in "Theory and practice of combinatorics," North-Holland, Amsterdam-New York, 1982, pp. 129-140. MR 86k:52015

[12] N. Robertson, D. Sanders, P. Seymour, R. Thomas, The four-colour theorem, J. Combin. Theory Ser. B 70 (1997) 2-44. MR 98c:05065

[13] Zh.-X. He, O. Schramm, Hyperbolic and parabolic packings, Discrete Comput. Geom. 14 (1995) 123-149. MR 96h:52017 
[14] O. Häggström, J. Jonasson, R. Lyons, Explicit isoperimetric constants, phase transitions in the random-cluster, and Bernoullicity, preprint, 2001.

[15] A. Kotzig, On the theory of Euler polyhedra (in Russian), Mat.-Fyz. Cas. Sloven. Akad. Vied 13 (1963) 20-31. MR 28:5375

[16] R. Lyons, Y. Peres, Probability on trees and networks, book manuscript, 2000.

[17] B. Mohar, Isoperimetric inequalities, growth, and the spectrum of graphs, Linear Algebra Appl. 103 (1988) 119-131. MR 89k:05071

[18] B. Mohar, Some relations between analytic and geometric properties of infinite graphs, Discrete Math. 95 (1991) 193-219. MR 93c:05113

[19] B. Mohar, Isoperimetric numbers and spectral radius of some infinite planar graphs, Math. Slovaca 42 (1992) 411-425. MR 94a:05003

[20] P. M. Soardi, Recurrence and transience of the edge graph of a tiling of the Euclidean plane, Math. Ann. 287 (1990) 613-626. MR 92b:52044

[21] T. Stehling, Über das Kotziggewicht normaler Pflasterungen, Resultate Math. 18 (1990) 347354. MR 92a:52029

[22] W. Woess, A note on tilings and strong isoperimetric inequality, Math. Proc. Cambridge Philos. Soc. 124 (1998) 385-393. MR 99f:52026

[23] A. Žuk, On the norms of the random walks on planar graphs, Ann. Inst. Fourier (Grenoble) 47 (1997) 1463-1490. MR 99g:60127

Department of Mathematics, University of Ljubljana, 1111 Ljubluana, Slovenia

E-mail address: bojan.mohar@uni-lj.si 